\section{Albumin coated liposomes: a novel platform for macrophage specific drug delivery}

\author{
Clément Vuarchey, Sushil Kumar, Reto \\ Schwendener \\ Institute of Molecular Cancer Research, \\ Laboratory of Liposome Research, \\ University of Zurich, Switzerland
}

\begin{abstract}
Here we report a new and efficient approach of macrophage specific drug delivery by coating liposomes with albumin. Activated albumin was reacted with liposomes containing polyethylene glycol (PEG) as hydrophilic spacers to create a flexible layer of covalently bound albumin molecules on the liposome surface. Albumin coated liposomes were taken up faster and more efficiently than uncoated liposomes by murine macrophages. Liposome uptake was significantly higher in macrophages as compared to other cell types tested (endothelial cells, fibroblasts, tumor cells), suggesting specificity for macrophages. In vivo, splenic macrophages phagocytosed BSA coated liposomes (BSA-L) at faster rates compared to conventional liposomes (L) and PEG liposomes (PEG-L). To prove the effectiveness of this new macrophage specific drug carrier, the bisphosphonates clodronate and zoledronate were encapsulated in BSA-L and compared with conventional liposomes. In vitro, treatment of macrophages with clodronate or zoledronate in BSA-L led to cytotoxic activity within a very short time and to up to 50 -fold reduced $\mathrm{IC}_{50}$ concentrations. In vivo, clodronate encapsulated in BSA-L depleted splenic macrophages at a 5-fold lower concentration as conventional clodronate-liposomes. Our results highlight the pharmaceutical benefits of albumin-coated liposomes for macrophage specific drug delivery.
\end{abstract}

\section{Introduction}

Cells with phagocytic properties have been subject of investigation since their discovery by Metchnikoff more than a decade ago. ${ }^{1}$ However, only the advent of modern technologies has made it possible to recognize the diverse roles macrophages play in an organism. Besides their homeostatic properties macrophages are the gate keepers of the immune system and they recognize and eliminate senescent and abnormal cells and generate signals that influence growth, differentia- tion and death of cells. ${ }^{1-3}$ Several disorders like arthritis, atherosclerosis, asthma, inflammatory bowel disease and others origin from the pathological activity of macrophages, conditions also considered as chronic inflammation. ${ }^{4-8}$ Microorganisms such as $M$. tuberculosis, ${ }^{9,10}$ parasites $^{11,12}$ and viruses such as HIV ${ }^{13-16}$ take advantage of macrophages as safe haven or powerful allies. In cancer, tumor associated macrophages contribute considerably to tumor growth and disease severity in many solid tumors. ${ }^{17-19}$ Given the central role macrophages play in this variety of human diseases, effective targeting of drugs to these cells could be an astute strategy for efficacious prevention and treatment of infectious and inflammatory diseases and cancer.

A multitude of options to deliver drugs to macrophages have been developed in the past. ${ }^{20}$ Most extensively studied are liposomes - nanosized unilamellar phospholipid bilayer vesicles. $^{21,22}$ Consequently, many drugs involved in macrophage-associated disorders have been studied using liposomes as carrier.

One of the most frequently reported application of macrophage specific liposome-mediated drug delivery are clodronate-liposomes that are successfully used to suppress macrophage activity by their depletion in various models of autoimmune diseases, transplantation, neurological disorders, viral and bacterial infections and cancer. Surprisingly, although effective in macrophage depletion, these liposomes have not been optimized for specific macrophage uptake, as they are composed of phosphatidylcholine and cholesterol, carry neutral charges and are either multilamellar or small unilamellar vesicles. ${ }^{23-26}$ Hence, to enhance macrophage uptake, we introduced a non-specific modification by linking albumin to liposomes. Liposomes are recognized by macrophages by their opsonization, which is a process where serum proteins, in particular proteins of the complement system, attach to the liposome surfaces to facilitate recognition and phagocytosis. ${ }^{27-33}$ Therefore, we reasoned that proteincoated and thus negatively charged liposomes would be phagocytosed more efficiently than conventional uncharged liposomes. We achieved this artificial opsonization by covalent attachment of albumin to the distal end of flexible poly (ethylene glycol) modified liposomes. Here we provide first proof of principle that albumin coated small unilamellar liposomes are a highly efficient drug delivery system for macrophages. Using fluorescence based approaches we noticed massive increase in uptake by macrophages when compared with conventional liposomes and with other cell types. Consequently, encapsulation of bisphosphonates in albumin coated liposomes led to a significant decrease of the cytotoxic dose and to more effective macrophage depletion in vivo.
Correspondence: Reto Schwendener, Institute of Molecular Cancer Research, University of Zurich, Winterthurerstr. 190, CH8057 Zurich, Switzerland.

Tel. +41.44.635 3483 - Fax: +41.44.635 3484 .

E-mail: rschwendener@imcr.uzh.ch

Key words: albumin coated liposomes, macrophage, specific targeting, drug delivery, bisphosphonates.

Acknowledgment: this work was supported in part by Novartis, Basel, Switzerland. The authors wish to thank Dr. Stefanie Krämer for help with the zeta-potential measurements.

Contributions: RS, CV, SK, study conception and manuscript writing; CV, SK, experiments and data analysis; SK, manuscript revising.

Conflict of interest: the authors report no conflicts of interest.

Received for publication: 10 May 2011. Accepted for publication: 18 July 2011.

This work is licensed under a Creative Commons Attribution NonCommercial 3.0 License (CC BYNC 3.0).

(C) Copyright C. Vuarchey et al., 2011

Licensee PAGEPress, Italy

Nanotechnology Development 2011; 1:e2

doi:10.4081/nd.2011.e2

\section{Materials and Methods}

\section{Chemicals}

Soy phosphatidylcholine (SPC) was from L. Meyer, Hamburg, GE and 1.2-distearoyl-snglycero-3-phosphoethanolamine-N-(maleimide (polyethylene glycol)2000) (DSPE-PEGMi) from Avanti Polar Lipids (Alabaster, Alabama, USA). Cholesterol (Chol), human serum albumin (HSA), N-ethylmaleimide and cysteine were from Fluka, Switzerland. D,L $\alpha$ tocopherol ( $\alpha$-toc) and mannitol from Merck (Darmstadt, Germany). 1,1'-dioctadecyl3,3,3',3'-tetramethyl-indocarbocyanine perchlorate (DiI) was from Molecular Probes (Invitrogen, Carlsbad, CA, USA). Hydroxylamine chloride, murine (MSA) and bovine serum albumin (BSA) were from SigmaAldrich (St. Louis, MO, USA). Clodronate was a gift from Farchemia SrL (Treviglio, Italy) and zoledronate a gift from Novartis International (Basel, Switzerland). N-succinimidyl-S-acetyl-thioacetate (SATA) was prepared as described. ${ }^{29}$

\section{Liposome preparation}

All liposomes were prepared by freeze-thawing and high pressure extrusion as 
described. ${ }^{24}$ Lipid compositions were as follows: (i) Conventional liposomes (L), SPC 104 $\mu \mathrm{mol} / \mathrm{mL}(80 \mathrm{mg} / \mathrm{mL})$, Chol $20 \mu \mathrm{mol} / \mathrm{mL}(8$ $\mathrm{mg} / \mathrm{mL}), \alpha$-toc $1.04 \mu \mathrm{mol} / \mathrm{mL}(0.45 \mathrm{mg} / \mathrm{mL})$; (ii) PEG-liposomes (PEG-L) and albumin coated liposomes (BSA-L, MSA-L, HSA-L), SPC 104 $\mu \mathrm{mol} / \mathrm{mL}$, Chol $20 \mu \mathrm{mol} / \mathrm{mL}, \alpha$-toc 1.04 $\mu \mathrm{mol} / \mathrm{mL}$, DSPE-PEG-Mi $2.1 \mu \mathrm{mol} / \mathrm{mL} \quad(6$ $\mathrm{mg} / \mathrm{mL}$ ); (iii) Conventional clodronate (CL) liposomes, SPC $130 \mu \mathrm{mol} / \mathrm{mL}(100 \mathrm{mg} / \mathrm{mL})$, Chol $25 \mu \mathrm{mol} / \mathrm{mL}(10 \mathrm{mg} / \mathrm{mL})$, $\alpha$-toc 1.3 $\mu \mathrm{mol} / \mathrm{mL}(0.56 \mathrm{mg} / \mathrm{mL})$ and (iv) PEG clodronate (PEG-CL) liposomes and BSA coated clodronate (BSA-CL) liposomes, SPC 130 $\mu \mathrm{mol} / \mathrm{mL}$, Chol $25 \mu \mathrm{mol} / \mathrm{mL}, \quad \alpha$-Toc 1.3 $\mu \mathrm{mol} / \mathrm{mL}$, DSPE-PEG-Mi $2.6 \mu \mathrm{mol} / \mathrm{mL} \quad(7.3$ $\mathrm{mg} / \mathrm{mL}$ ). For fluorescent liposomes DiI was added at $0.35 \mu \mathrm{mol} / \mathrm{mL}(0.3 \mathrm{mg} / \mathrm{mL})$.

Lipids were dissolved in methanol/methylene chloride (1:1, v/v) and solvents removed by evaporation at $40^{\circ} \mathrm{C}$ (Rotavapor, Büchi Labortechnik AG, Flawil, Switzerland). Dried lipid films were dispersed in phosphate-mannitol buffer (PB-Man, $20 \mathrm{mM}$ phosphate, 230 $\mathrm{mM}$ mannitol, $\mathrm{pH}$ 7.4), supplemented with $66.25 \mathrm{mg} / \mathrm{mL}$ clodronate or $4 \mathrm{mg} / \mathrm{mL}$ zoledronate for the bisphosphonate liposomes. Liposomes were obtained by three cycles of freeze-thawing in liquid nitrogen and $40^{\circ} \mathrm{C}$ water, followed by repetitive extrusion through NucleporeTM membranes (Sterico AG, Dietikon, Switzerland) of $400 \mathrm{~nm}(3 \mathrm{x})$ and $100 \mathrm{~nm}(8 \mathrm{x})$ pore size using a Lipex ${ }^{\mathrm{TM}}$ extruder (Northern Lipids Inc., Burnaby, BC, Canada). For the clodronate liposomes (3 and 4) extrusion was done with $400 \mathrm{~nm}$ filters (8x) and non-encapsulated biphosphonate was removed by extensive dialysis (Spectrapore tube, 12-14 kD mol.wt. cut-off) using PB-Man as dialysis buffer $(1: 100, \mathrm{v} / \mathrm{v})$. All preparations were sterile filtrated $(0.45 \mu \mathrm{m}$ Millex-HV filters, Millipore, Billerica, MA, USA). Liposome size and homogeneity were measured by dynamic laser light scattering (Nicomp 370, Nicomp Corp., Santa Barbara, CA, USA). The $\zeta$-potential was determined with a Malvern Zetasizer 3000 HAS (Malvern Instruments, Malvern, UK) and bisphosphonate encapsulation assessed by liquid scintillation counting (Tri-Carb, Canberra Packard Int., Zurich, Switzerland).

\section{Albumin coupling}

Albumins were coupled to the liposomes as described. $^{34}$ Thiolation was performed by modification of earlier described methods. ${ }^{35}$ SATA (1 mg) dissolved in dimethylformamide (100 $\mu \mathrm{L})$ was added per ml albumin solution (10 $\mathrm{mg} / \mathrm{mL}$ ) and incubated $1 \mathrm{~h}$ at RT in HEPES buffer (10 mM HEPES, $145 \mathrm{mM} \mathrm{NaCl,} 2 \mathrm{mM}$ EDTA, pH 7.4). Unreacted SATA was removed by dialysis (Spectrapore tube, 12-14 kD cutoff) at $4^{\circ} \mathrm{C}$ overnight. Thioacetylated albumin was deacetylated by addition of hydroxylamine
(1M, $100 \mu \mathrm{L} / \mathrm{mL}$ BSA, $30 \mathrm{~min}$ ). Albumin coupling to freshly prepared liposomes was done by incubation at equal volumes at RT for $8 \mathrm{~h}$. Addition of N-ethylmaleimide $(1: 60 \mathrm{~mol} / \mathrm{mol})$ stopped the reaction. Unreacted maleimide residues were blocked by addition of excess cysteine as described before. ${ }^{36}$ Unbound albumin was removed by gel filtration on Sephadex G-100 columns. Protein content was measured using the Bradford assay according to the manufacturer's instructions (Bio-Rad Laboratories GmbH, Munich, Germany).

\section{Cell lines and cell culture}

Murine macrophages (RAW 247.6, ATCC TIB-71) and Lewis lung carcinoma (LLC, ATCC CRL-1642) cells were cultivated in RPMI 1640 with L-glutamine (10\% FBS, 10'000 U/mL penicillin, $10 \mathrm{mg} / \mathrm{mL}$ streptomycin). Murine NIH 3T3 fibroblasts and pancreatic islet endothelial MS1 (ATCC CRL-2279) cells were cultivated in DMEM with $4.5 \mathrm{mg}$ glucose/L (10\% FBS and antibiotics). All cells were maintained at $37^{\circ} \mathrm{C} / 5 \% \mathrm{CO}^{2}$ in a humidified incubator.

\section{Flow cytometry and fluorescence microscopy}

Cells were seeded at a density of 25,000 cells per well in 24-well plates and grown in RPMI medium for $48 \mathrm{~h}$. Cells were incubated with medium either containing: (i) DiIlabeled L; (ii) DiI-labeled PEG-L or (iii) DiIlabeled BSA-L at concentrations of 10 (8 ng), 50 (20 ng) or 250 (200 ng) nmol SPC/mL liposomes in $300 \mu \mathrm{L}$ medium for different incubation times ( $10 \mathrm{~min}, 30 \mathrm{~min}, 1 \mathrm{~h}, 3 \mathrm{~h}$ and $24 \mathrm{~h}$ ). Then, cells were washed three times with PBS. For macrophages, the cell layer was gently scraped in PBS using a cell scraper to facilitate detachment of the cells. For the other cell types, cells were harvested by trypsinization and washed once with PBS. Cells were analyzed by flow cytometry (CyAn 9 ADP Beckman Coulter, Nyon, Switzerland) to distinguish the uptake between the three types of liposomes. Cells were measured for DiI fluorescence and forward and side scatter were used to gate living cells. Liposome uptake was calculated by dividing the mean log of Dil fluorescence of liposome-treated viable cells by the mean log of DiI fluorescence of untreated control cells. For fluorescence microscopy cells were washed in PBS after the indicated incubation times, fixed with formaldehyde (3\% in PBS, AppliChem GmbH, Darmstadt, Germany) and incubated with DAPI $(2 \mu \mathrm{g} / \mathrm{mL}$ in PBS, Roche Diagnostics GmbH, Mannheim, Germany) for $10 \mathrm{~min}$ in order to stain the cell nuclei. The uptake of liposomes to the different cell types was examined using an Olympus OBS IX81 microscope (Olympus, Tokyo, Japan).

\section{Cell viability}

Cell viability was evaluated using the resazurin method. ${ }^{37}$ Cells (25,000/well) in 24 well plates were grown for $30 \mathrm{~h}$. Medium containing increasing concentrations of 1-1000 $\mu \mathrm{M}$ for CL, PEG-CL and BSA-CL or 0.1-10 $\mu \mathrm{M}$ for ZL, PEG-ZL and BSA-ZL was added for $1 \mathrm{~h}$, $3 \mathrm{~h}, 24 \mathrm{~h}$ or $48 \mathrm{~h}$. Then, cells were washed $3 \mathrm{x}$ with PBS and the $1 \mathrm{~h}$ and $3 \mathrm{~h}$ treatments were additionally incubated with RPMI for $23 \mathrm{~h}$ or $21 \mathrm{~h}$, respectively, to allow the bisphosphonates to take effect. Resazurin $(0.5 \mathrm{~mL}$ in medium) was added and after $4 \mathrm{~h}$ incubation at $37^{\circ} \mathrm{C}$ fluorescence was measured at $590 \mathrm{~nm}$ emission with $540 \mathrm{~nm}$ excitation wavelength in a SpectraMax M5/M5e Reader (Molecular Devices Corp., Sunnyvale, CA, USA). Cytotoxicity $\left(\mathrm{IC}_{50}\right.$-values) was determined by graphic extrapolation by plotting drug concentration against percentage of viable cells and taking untreated cells as $100 \%$ viability. Empty liposomes were not toxic to cells (data not shown). All measurements were carried out twice in duplicates.

\section{Animal studies}

Analysis of macrophage depletion in spleens was done in C57Bl/6 mice (3/group) by i.v. injection of $50 \mu \mathrm{L}$ of Dil-labeled L or BSA-L. After 1.5 or $6 \mathrm{~h}$ the spleens were removed and immersed in ice cold DMEM (10\% FBS, $4.5 \mathrm{mg}$ glucose/L, 1\% antibiotics) followed by mashing on a $70 \mu \mathrm{m}$ cell strainer (Becton Dickinson Labware, Le Pont de Claix, France) to create a single cell suspension. After centrifugation (2000 rpm, $5 \mathrm{~min}$ ) the pellet was resuspended and filtrated (35 $\mu \mathrm{m}$ nylon mesh filter, Beckton Dickinson), followed by 10 min treatment with red blood cell lysis buffer (Pharm Lyse ${ }^{\mathrm{TM}}$, BD Pharmingen, San Diego, CA, USA), centrifuged and re-suspended in FACS buffer (2\% FCS in PBS). The CD16/32 Fc blocking antibody (1:300, v/v, BioLegend, San Diego, CA, USA) was added to decrease the background signal. Cells were incubated with the F4/80-APC antibody (1:200 in FACS buffer) for 30 min on ice and immediately analyzed (CyAn $\left.{ }^{\mathrm{TM}} 9 \mathrm{ADP}\right)$. To study depletion of macrophages, $\mathrm{C} 57 \mathrm{Bl} / 6$ mice (3/group) received 0.2 or $1 \mathrm{mg}$ CL or BSA-CL by i.p. injection or empty BSA-L as controls. After $24 \mathrm{~h}$ the spleens were removed and cell suspensions obtained as described above. Percentage of macrophage depletion was determined by flow cytometry of F4/80 stained cells.

\section{Statistical analysis}

Comparisons among different liposome preparations were made by the unpaired Student's t-test using the GraphPad Prism 5.03 software. Differences were termed statistically significant at $\mathrm{P}<0.05$ and data expressed as mean \pm SD. 


\section{Results}

A schematic representation of conventional liposomes L, poly(ethylene glycol) liposomes PEG-L and albumin coated liposomes BSA-L is shown in Figure 1.

The structural changes of BSA coated liposomes are summarized in Table 1 . The mean diameter of $\mathrm{L}$ was $96.8 \pm 1.7 \mathrm{~nm}$ with a nearly neutral $\zeta$-potential. Addition of DSPEPEG2000-Mi at $2 \mathrm{~mol} \%$, - in contrast to 7.5 mol\% used for conventional long circulating PEG-liposomes ${ }^{38}$ - increased the diameter by $10 \mathrm{~nm}$ and decreased the $\zeta$-potential by $5 \mathrm{mV}$ as reported previously. ${ }^{38}$

Covalent coupling of BSA to PEG-L increased liposome size by about $40 \mathrm{~nm}$ and decreased the $\zeta$-potential to $-10 \mathrm{mV}$, confirming that negatively charged albumin was attached on the outer liposome surface. Liposome size changed slightly after storage up to 60 days at $4^{\circ} \mathrm{C}$ (Table 1). Cell incubation results were identical with liposomes stored for 60 days, indicating good liposome stability (data not shown). The observed size increase of PEG-L is the direct consequence of pegylation, which leads to a coating thickness of $5 \mathrm{~nm} \cdot{ }^{38}$ BSA molecules are negatively charged at physiological $\mathrm{pH}$ and have either a heart-like shape, which can be approximated by a triangular shape ${ }^{39}$ a prolate ellipsoid ${ }^{40}$ or an oblate ellipsoid, ${ }^{41}$ depending on the method of structure analysis used. Calculation of the BSA concentration on the liposome surface based on the data obtained from the Bradford protein assay resulted in an average of $64 \pm 3 \mu \mathrm{g} \mathrm{BSA} / \mu \mathrm{mol}$ total lipids, which corresponds to 100-130 BSA molecules attached to one liposome calculated as decribed. ${ }^{36}$ These results are in accordance with those reported by Thöle et al. ${ }^{42}$

Encapsulation of the bisphosphonates resulted in similar sizes as observed with empty liposomes (Table 2). The encapsulation rate of $30-33 \%(18 \pm 2.5 \mathrm{mg} / \mathrm{mL}$ for clodronate and $1.25 \pm 0.3 \mathrm{mg}$ for zoledronate) was determined by radioactive trace labeling (data not shown). Here, it is noteworthy that the mean diameter of clodronate liposomes after repetitive extrusion through $400 \mathrm{~nm}$ membranes resulted in a range of $187 \pm 88 \mathrm{~nm}$ which is considerably smaller than expected. Clodronate probably interacts with the lipid bilayer in a way that a condensation effect occurs. To study the preferential uptake RAW macrophages were treated with DiI-labeled L, PEG-L and BSA-L and subsequently analyzed by fluorescence microscopy and flow cytometry. As shown in Figure 2, BSA-L were taken up more efficiently by the macrophages in a time and concentration dependent manner. At each time point the amount of BSA-L taken up was significantly higher than that of L and PEG-L. Importantly, there was no difference in uptake between BSA-L, HSA-L, and MSA-L (Figure 2A, C) suggesting that the higher uptake of albumin coated liposomes was not due to the antigenicity of a foreign protein (BSA or HSA on murine macrophages) but rather due to the albumin coating. This permits to choose the albumin independently of the species studied, without provoking immune responses that could be induced by antigenic albumin. Consequently, for the following experiments, BSA-L were used.

The flow cytometry results (Figure 2D) showed that macrophage uptake of BSA-L was about 17-times higher after $1 \mathrm{~h}$ incubation and remained 4-times higher after $24 \mathrm{~h}$ as compared to L. Compared to PEG-L uptake of BSA$\mathrm{L}$ was 53 -times higher after $1 \mathrm{~h}$ incubation and 9 times higher after $24 \mathrm{~h}$ incubation. These differences are concentration independent since no change in uptake was observed at liposome concentrations of 10 and $50 \mathrm{nmol}$ $\mathrm{SPC} / \mathrm{mL}$ (data not shown). The results suggest that macrophages phagocytose BSA-L at faster

rates than the control liposomes $\mathrm{L}$ and PEG-L. Another aspect highlighted by these results is that PEG-L uptake by macrophages is significantly lower as compared to L, confirming earlier findings. ${ }^{43}$

Examination of macrophages exposed to DiI-labeled BSA-L at a higher magnification (Figure 2B) revealed that DiI was uniformly distributed on the surface and in the cytoplasm of the macrophages suggesting that higher uptake is due to stronger surface binding of BSA-L and higher phagocytic activity. To evaluate the specificity of BSA-L towards macrophages, three other cell lines were analyzed in a comparable set of experiments; cancer cells (Lewis lung carcinoma, LLC), fibroblasts (NIH 3T3) and endothelial cells (MS-1). Uptake of DiI-labeled liposomes was significantly lower in these cells when compared to the macrophages (Figure 3). After $3 \mathrm{~h}$ or $24 \mathrm{~h}$ incubation DiI-labeled BSA-L uptake increased on macrophages between 40 - and 220 -fold (Figure 2D), compared to a significantly lower

Table 1. Liposome size and $\zeta$-potential of L, PEG-L and BSA-L. ( $n=3$ for mean particle size and $n=5$ for $\zeta$-potential measurements).

\begin{tabular}{lcccc} 
Liposome & $\begin{array}{c}\text { Mean } \\
\text { diameter }(\mathrm{nm})\end{array}$ & $\begin{array}{c}\text { Mean diameter } \\
\text { after } 30 \text { days }(\mathrm{nm})\end{array}$ & $\begin{array}{c}\text { Mean diameter } \\
\text { after } 60 \text { days }(\mathrm{nm}) \text { potential }(\mathrm{mV})\end{array}$ \\
$\mathrm{L}$ & $96.8 \pm 7$ & $98.2 \pm 2.2$ & $97.6 \pm 2.8$ & $-1 \pm 0.6$ \\
$\mathrm{PEG}-\mathrm{L}$ & $111.3 \pm 59$ & $110.6 \pm 2.6$ & $111.5 \pm 2.5$ & $-5.5 \pm 0.7$ \\
\hline BSA-L & $151.1 \pm 01$ & $153.3 \pm 5.9$ & $158.5 \pm 3.9$ & $-10.7 \pm .4$ \\
\hline
\end{tabular}

Table 2. Liposome size, encapsulation rate and cytotoxicity $\left(\mathrm{IC}_{50}\right.$ in $\mu \mathrm{M}$, see also Figure 5) of bisphosphonates in liposomes $(n=3)$.

\begin{tabular}{lccccc} 
Liposome & $\begin{array}{c}\text { Mean diameter } \\
(\mathrm{nm})\end{array}$ & $\begin{array}{c}\text { Encapsulation } \\
\text { rate }(\%)\end{array}$ & $\begin{array}{c}\mathrm{IC}_{50} \\
\mathbf{3 ~ h}\end{array}$ & $\begin{array}{c}\mathrm{IC}_{50} \\
24 \mathrm{~h}\end{array}$ & $\begin{array}{c}\mathrm{IC}_{50} \\
\mathbf{4 8} \mathrm{h}\end{array}$ \\
CL & $186.5 \pm 88$ & $30.9 \pm 2.5$ & $\mathrm{nd}$ & $\mathrm{nd}$ & nd \\
PEG-CL & $184.2 \pm 52$ & $30.3 \pm 1.0$ & $\mathrm{nd}$ & $\mathrm{nr}$ & 620 \\
\hline BSA-CL & $215.8 \pm 80$ & $30.3 \pm 1.0$ & 200 & 40 & nd \\
ZL & $184.2 \pm 54$ & $33.7 \pm 1.4$ & $\mathrm{nr}$ & 50 & 4 \\
\hline PEG-ZL & $177.5 \pm 26$ & $32.8 \pm 0.3$ & $\mathrm{nr}$ & $>50$ & 5.6 \\
BSA-ZL & $213.7 \pm 97$ & $32.8 \pm 0.3$ & 3.6 & 1 & 0.225 \\
\hline
\end{tabular}

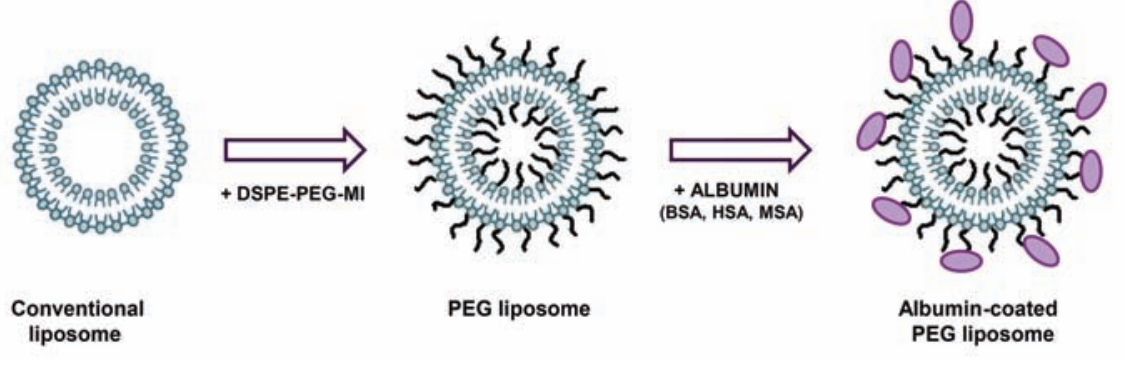

Figure 1. Schematic representation of conventional liposomes L, poly(ethylene glycol) liposomes PEG-L and albumin coated liposomes BSA-L. Albumin coating is achieved by the reaction of activated thiolated albumin with maleimide groups located at the terminal ends of the poly(ethyleneglycol) chains on the liposome surface. 

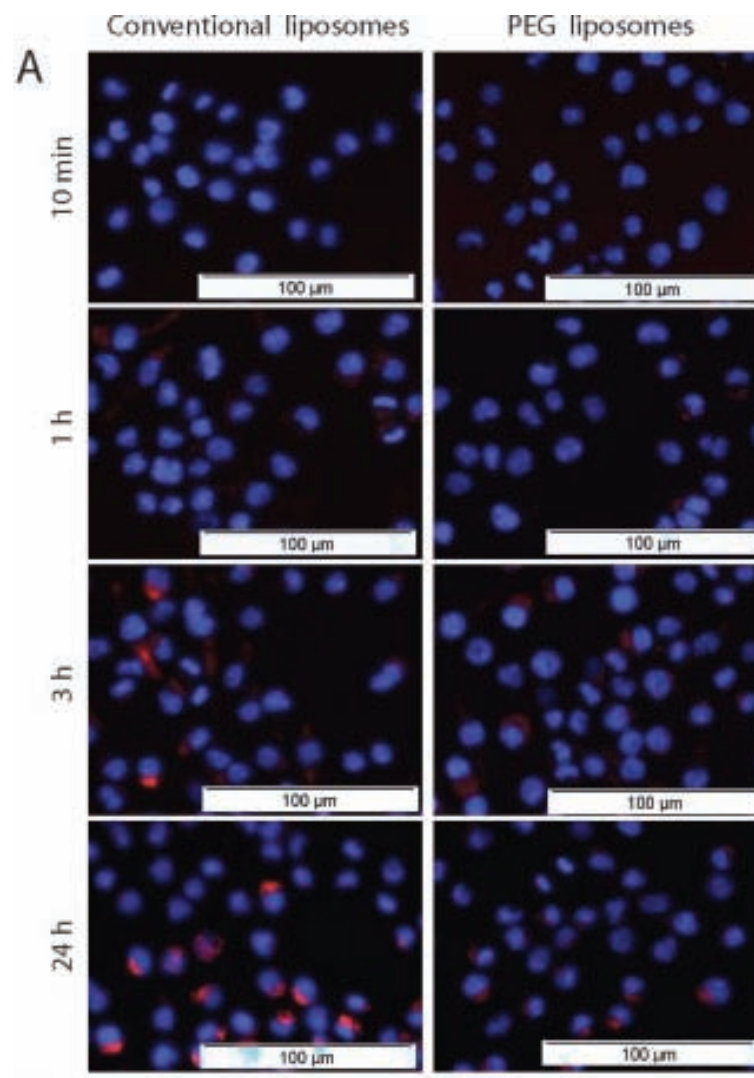

BSA coated PEG liposomes
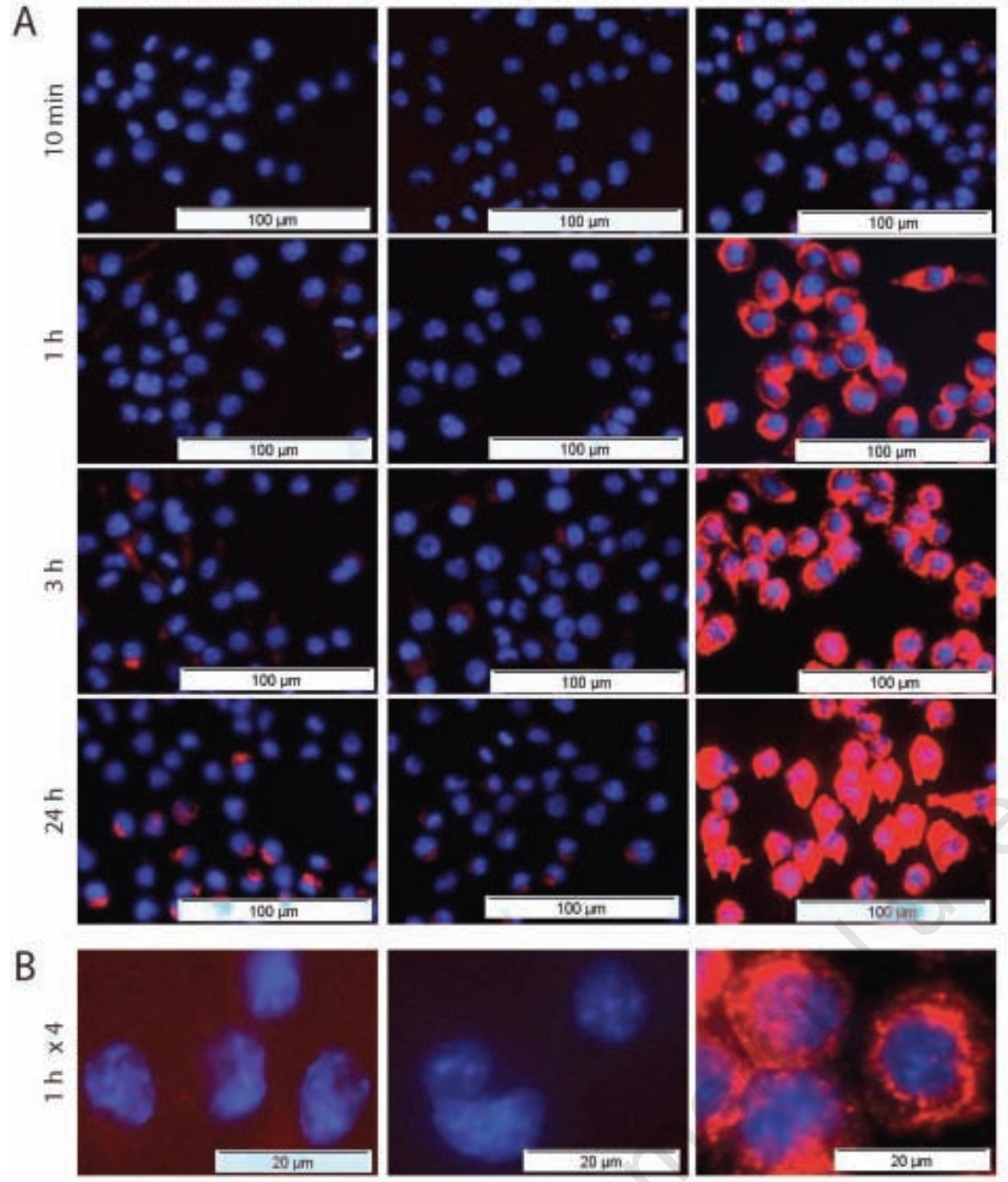

C HSA coated PEG liposomes

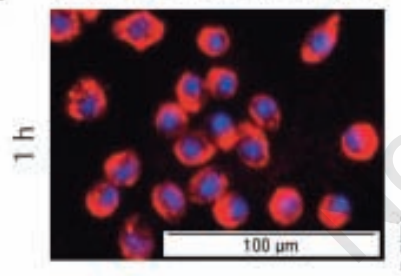

MSA coated PEG liposomes
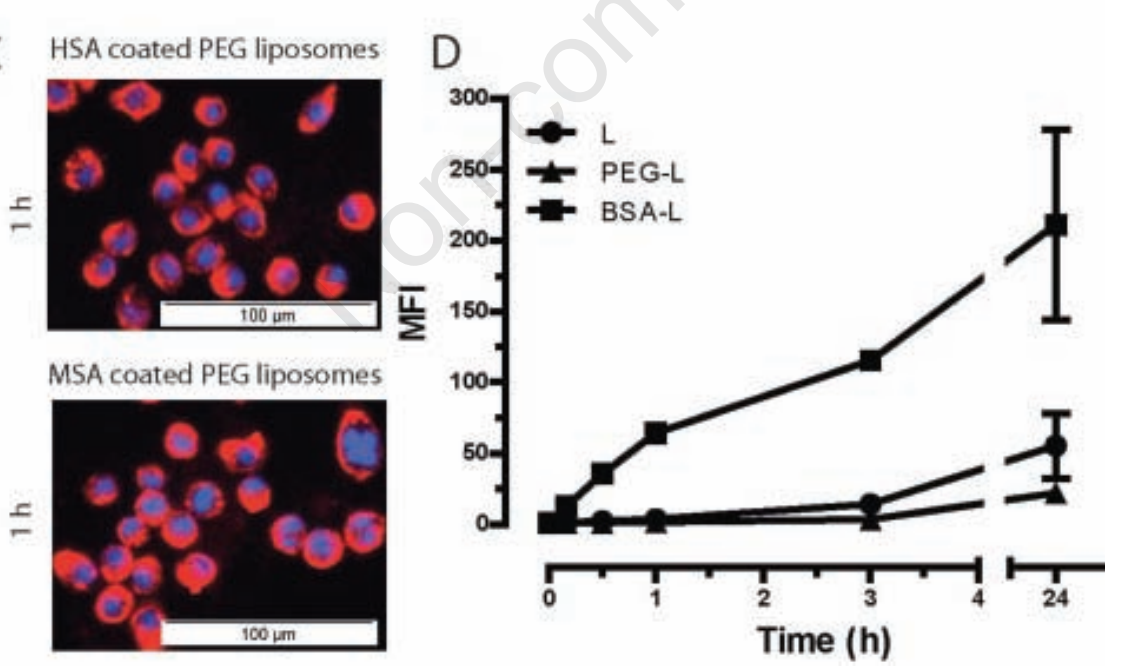

Figure 2. In vitro uptake of DiI-labeled liposomes by macrophages. (A) Fluorescence microscopy images of macrophages incubated with $250 \mathrm{nmol} \mathrm{SPC} / \mathrm{mL}$ DiI-labeled liposomes at different time points. (B) Uptake after $1 \mathrm{~h}$ incubation at higher magnification $(640 \mathrm{x})$. (C) Uptake of DiI-labeled HSA-L and MSA-L after 1h. Red is fluorescence of DiI and blue is fluorescence of DAPI. (D) Quantification of the cellular uptake of DiI-labeled liposomes by macrophages by flow cytometry (MFI, mean fluorescence intensity). Liposome uptake was calculated by dividing the mean $\log$ of DiI fluorescence of liposome-treated cells by the mean $\log$ of DiI fluorescence of untreated cells.
1- and 12-fold uptake for the other cells.

These results confirm the target specificity of the albumin-coated liposomes towards macrophages. When analyzed by flow cytometry slight differences of liposome uptake between the cell lines were observed, probably by virtue of the high sensitivity of the method. The flow cytometric analysis also showed low uptake of $L$ by any other cell line, in comparison to the macrophages which was further decreased when BSA-L and PEG-L were used (Figure 3).

Coating the liposome surface with inert, biocompatible polymers such as PEG interferes with the ability of liposomes to interact with target cells. ${ }^{44,45}$ Rather, such polymer coating at saturating concentrations is used to prolong the blood circulation time of liposomes. ${ }^{38,43-45}$ The evaluation of our results suggests that albumin coated liposomes have a 2 -fold advantage over uncoated liposomes, on one hand they are preferred nanoparticles for macrophages and on the other hand they show distinctly lower uptake by other cell types. The in vivo characteristics of the uptake of DiIlabeled L, PEG-L and BSA-L were assessed in spleens after intravenous injection (Figure 4). PEG-L were not used as control since several studies including ours showed that surfacegrafted PEG reduce opsonization leading to prolonged blood circulation and reduced macrophage uptake. ${ }^{43-45}$ The spleen is the peripheral reservoir of myeloid cells that is constantly replenished by bone marrow myeloid progenitors. ${ }^{2}$ The analysis of spleen cells revealed that there was no significant difference between BSA-L and L concerning the percentage of targeted macrophages as reflected by the comparable percent values of F4/80 positive cells ranging from 1.61-2.35\% of all viable splenocytes (Figure 4A, upper right quadrant). Rather, it seems that macrophages phagocytose albumin-grafted liposomes at a highly faster rate compared to other liposome types. Ninety minutes after liposome injection

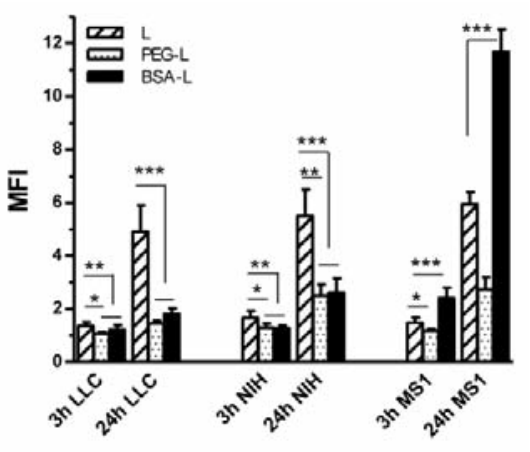

Figure 3. In vitro evaluation of the uptake of DiI-labeled liposomes by LLC, NIH 3T3 and MS-1 cells by flow cytometry. MFI, mean fluorescence intensity; *, $\mathbf{P}<0.05$; ${ }^{* *}, \mathbf{P}<0.01 ;{ }^{* * *}, \mathbf{P}<\mathbf{0 . 0 0 1}$. 
the population of $\mathrm{F} 4 / 80^{+}$macrophages which had taken up Dil-labeled liposomes had already reached $35.14 \%$ (Figure $4 \mathrm{~A}$, cells in box in upper right quadrant) with the BSA-L, while no such population was found after injection of L. After $6 \mathrm{~h}$, uptake of $\mathrm{L}$ had reached $6.1 \%$, whereas it had decreased from 35.14-27.5\% with BSA-L. This result indicates that $6 \mathrm{~h}$ after administration of BSA-L macrophages were already saturated while the rate of phagocytosis of L was still increasing. The decrease of the DiI-positive macrophage population after BSA-L treatment between 90 min and $6 \mathrm{~h}$ can possibly be explained by exocytosis of liposomes, whereas exchange of the lipophilic dye DiI between macrophages and other cells can be excluded due to the high membrane stability of the dye. ${ }^{46}$ The bar graph shown in Figure $4 \mathrm{~B}$ recapitulates the macrophage uptake results of three individual experiments. These data correlate well with the results from the in vitro studies and confirm the in vivo efficiency of BSA-L specifically targeting macrophages. Hence, we can assume that uptake of albumin coated liposomes in vivo by macrophages is faster and occurs at higher avidity compared to L. Higher uptake of BSA-L by macrophages is indicative of enhanced delivery of encapsulated drugs. Thus, we tested the cytotoxic effect of clodronate and zoledronate containing liposomes on macrophages. In Figure 5, doseresponse curves are presented and the extrapolated $\mathrm{IC}_{50}$-values are summarized in Table 2 . BSA-CL were highly toxic to macrophages showing effects already after $1 \mathrm{~h}$ and reaching an $\mathrm{IC}_{50}$ of $200 \mu \mathrm{M}$ after $3 \mathrm{~h}$. When compared to CL, the BSA-L were 15.5 times more effective in the $24 \mathrm{~h}$ assay. Correspondingly, BSA-ZL liposomes were 50 - and 25 -times more toxic after $24 \mathrm{~h}$ and $48 \mathrm{~h}$, respectively. The effect of the zoledronate-liposomes is comparable to results reported by Shmeeda et al. describing strong cytotoxic effects of folate targeted liposomes on various cell types expressing folate receptors at similar $\mathrm{IC}_{50}$ concentrations. ${ }^{47}$

Thus, our results show that BSA-L deliver the encapsulated bisphosphonates at faster rates and more efficiently to macrophages, causing cytotoxic effects at shorter incubation times as compared to controls. This is particularly important in the case of conventional clodronate-liposomes, since the quantity of the administered drug to deplete macrophages is considerable and in the range of the $\mathrm{LD}_{50}$ for mice (5.54 mM or $2 \mathrm{mg} / 20 \mathrm{~g}$ mouse weight). ${ }^{24}$ CL are widely used to deplete macrophages..$^{23-26}$ Therefore, we tested the macrophage-depleting efficacy of BSA-CL in mouse spleens and compared it with CL. Application of $1 \mathrm{mg}$ of BSA-CL caused $67.6 \pm 3.5 \%$ depletion of the $\mathrm{F} 4 / 80^{+}$population of spleen macrophages after $24 \mathrm{~h}$, whereas after CL treatment depletion reached only $36.2 \pm 9.4 \%$. At $0.2 \mathrm{mg}$ the depletion efficiency of BSA-CL $(43.7 \pm 3.5 \%)$ was similar to that of $1 \mathrm{mg}$ CL (Figure 6). These results further confirm that BSA-L are not only preferred for phagocytosis by macrophages but that they also deliver drugs more efficiently and consequently deplete macrophages at lower clodronate concentrations.

\section{Discussion}

In this study we prepared albumin-coated liposomes with remarkably increased uptake properties on macrophages compared to conventional liposomes. Such macrophage specific liposomes represent an interesting platform to transport drugs or other compounds efficiently to macrophages, thus opening new possibilities to treat diseases where macrophages are involved. Liposomes are mainly recognized by macrophages equally as other nanoparticulate carriers and pathogens by their opsonization, a process where serum proteins, in particular those of the comple-
A

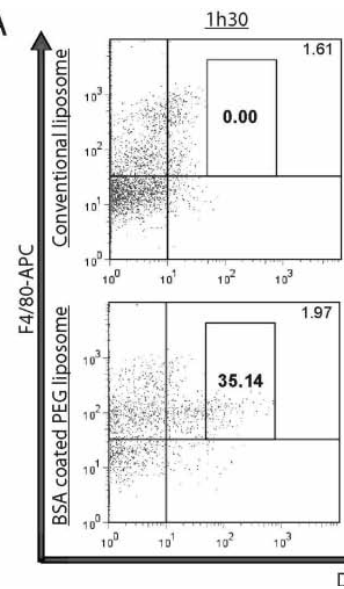

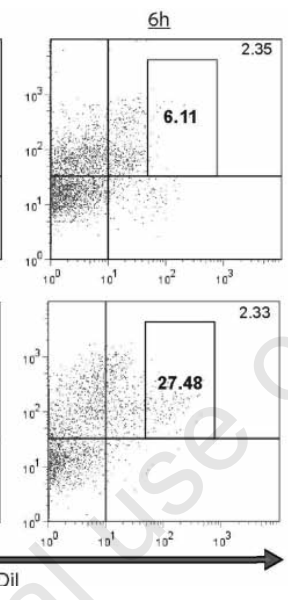

B

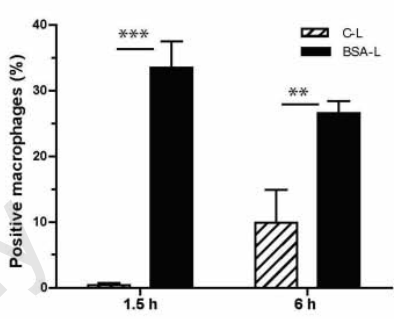

Figure 4. In vivo evaluation of the cellular uptake of DiI-labeled liposomes by spleen macrophages by flow cytometry. In A, a representative logarithmic plot of $\mathrm{F} 4 / \mathrm{80}^{+}$ macrophages (F4/80-APC) versus DiI-labeled liposomes (DiI) is shown. B summarizes the mean percentage of $\mathrm{F} 4 / \mathbf{8 0}^{+}$macrophages which had a 5 times higher DiI fluorescence intensity (cells in box in upper right quadrant in A) compared to all macrophages selected in the upper right quadrant. DiI-liposome uptake was quantified by determining the percentage of fluorescent viable macrophages. Non specific fluorescence was eliminated by gating the signal of non treated mice $(\log \mathrm{PE}=10)$ and percentages of $\mathrm{F} 4 / 80^{+}$ macrophages were determined by eliminating the signal of unstained samples. The uptake of the two types of liposomes by macrophages was evaluated by gating the $\mathrm{F} 4 / 80^{+}$ macrophages, which had a 5-fold higher fluorescence $(\log P E=50)$ than all positive fluorescent macrophages. The bars represent the mean percentage of viable macrophages $\pm S D$, $\mathrm{n}=3$; ${ }^{* *}, \mathbf{P}<0.01 ;{ }^{* * *}, \mathbf{P}<0.001$.

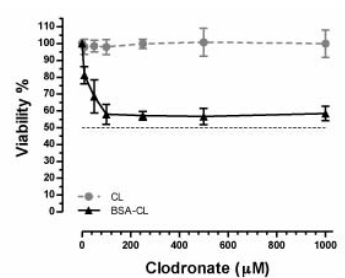

$1 \mathrm{~h}$

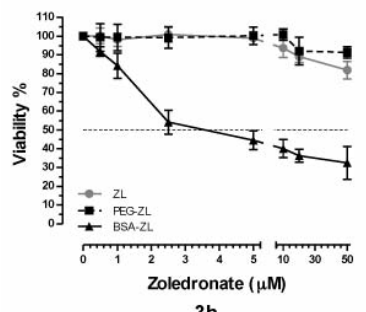

$3 \mathrm{~h}$

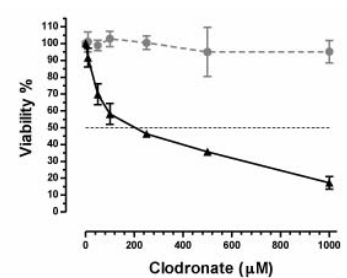

$3 \mathrm{~h}$

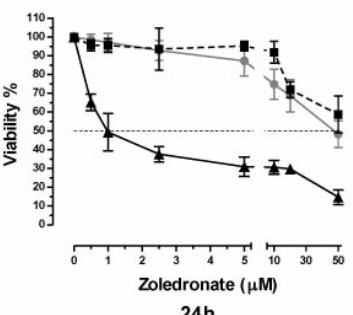

$24 \mathrm{~h}$

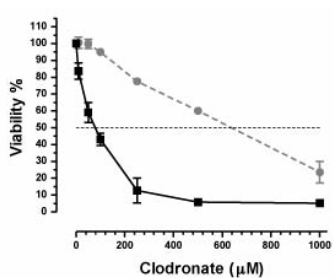

$24 \mathrm{~h}$

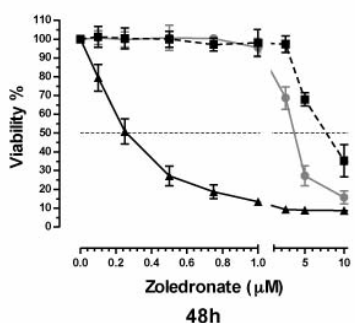

$48 \mathrm{~h}$

Figure 5. Viability of macrophages after treatment with CL and BSA-CL (upper panel) and ZL, PEG-ZL and BSA-ZL (lower panel) after $1 \mathrm{~h}, 3 \mathrm{~h}$ and $24 \mathrm{~h}$ or $48 \mathrm{~h}$ drug incubation. 


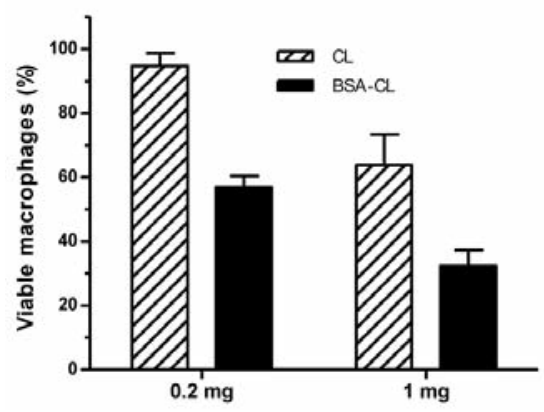

Figure 6. In vivo evaluation of the depletion of splenic macrophages with $C L$ and BSA-CL by flow cytometry $(n=3)$. To study the depletion of macrophages $\mathrm{C} 57 \mathrm{Bl} / 6$ mice $(n=3)$ were treated by intraperitoneal injection of 0.2 or $1 \mathrm{mg}$ of CL or BSA-CL or an equivalent number of empty BSA-L as controls. After $24 \mathrm{~h}$ the spleen was removed and cell suspensions were obtained. Macrophages were identified by gating the F4/80-APC channel as described in Figure 4.

ment system, attach to their surfaces to facilitate recognition and phagocytosis. ${ }^{27-33}$ Moreover, the degree of liposome binding and subsequent ingestion by macrophages depends on a number of factors including lipid composition, vesicle type, size and surface properties. Small unilamellar liposomes deliver drugs more effectively than larger uni- or multilamellar liposomes and charged liposomes associate more effectively to cells and deliver their content more efficiently than neutral liposomes..$^{21,22,48,49}$ Finally, coating liposome surfaces with proteins or cell surface specific ligands such as Fc-receptor, complement, folate, fibronectin, lipoproteins, mannosyl and galactosyl receptors and others significantly promote uptake of liposomal content by macrophages. ${ }^{21,22,50-55}$ However, many of these strategies have shortcomings such as system complexity, production costs and restricted applications and none of them has been routinely exploited to increase macrophage uptake of liposomes for therapeutic interventions. Since the modification of albumin with a bispecific-coupling molecule as SATA and its subsequent reaction with maleimide-modified PEG-lipids are straightforward reactions that occur in aqueous media, we argue that the preparation of albumin coated liposomes is an easy procedure. Potential disadvantages encountered with the preparation of larger batches remain to be investigated.

Therefore, it appears that enhancement of liposome binding and uptake by macrophages could be achieved by interference with protein adsorption and reactions with the complement system. It has been shown that neutral liposomes are poor activators of the complement system as compared to negatively charged vesicles. ${ }^{30,56}$ Besides charge, liposome size and concentration play also an important role in complement activation. . $^{28,57}$

Consequently, we reasoned that proteincoated and thus negatively charged unilamellar liposomes of intermediate sizes would be phagocytosed more efficiently than conventional liposomes. We achieved this artificial opsonization by covalent attachment of albumin to PEG-modified liposomes.

Albumin is constantly synthesized in the liver and its homeostasis is maintained by a balanced catabolism occurring in all tissues. Most albumin is degraded in muscle, liver and kidney. ${ }^{40}$ Albumin itself is not taken up by macrophages and it is only upon its covalent linking to nanoparticulate vesicles such as liposomes that macrophage uptake occurs. Thus, possible mechanisms that improve and accelerate the uptake of albumin coated liposomes are that the protein may accelerate opsonisation or that binding of the liposomes to the macrophage cell surface is facilitated and therefore phagocytosis occurs at higher rates as compared to uncoated liposomes. However, the exact mechanisms remain to be elucidated.

More recently, albumin-liposome conjugates have been studied in order to develop long circulating drug carriers. ${ }^{58,59}$ In these reports it was shown that introduction of albumin on the surface of conventional PEG-liposomes prolonged circulation in plasma compared to PEG-liposomes. However, in contrast to our formulations, in these studies albumin was directly grafted onto the lipid surface of liposomes containing PEG at the conventional amounts used for long circulating liposomes. Thus, the albumin molecules directly attached to the liposome surface were covered by the PEG chains, which might prevent or slow down liposome opsonization. In this study, bisphosphonates were used as prototype drugs, however it can be assumed that other drugs such as protein inhibitors or DNA and RNA (e.g. siRNA, microRNA and antimicroRNA) based therapeutics or nanozymes encapsulated in albumin-liposomes and targeted to macrophages will have similar pharmaceutical advantages. ${ }^{60-63}$

In summary, in this study a new nanodrug carrier platform for specific macrophage delivery, - albumin coated liposomes -, was developed and evaluated in vitro and in vivo. These surface coated liposomes are characterized by a significantly improved uptake and specificity towards macrophages, a lower uptake rate by non myeloid cell types and an enhanced accumulation in splenic macrophages, as compared to conventional and pegylated liposomes. Our results indicate that albumin coated liposomes represent a promising platform for macrophage specific drug delivery for the administration of bisphosphonates and other drugs and compounds.

\section{References}

1. Gordon S, Taylor PR. Monocyte and macrophage heterogeneity. Nat Rev Immunol 2005;5:953-64.

2. Taylor PR, Martinez-Pomares L, Stacey M, et al. Macrophage receptors and immune recognition. Annu Rev Immunol 2005;23: 901-44.

3. Gordon S. The macrophage: past, present and future. Eur J Immunol 2007;37 Suppl 1:S9-17.

4. Bondeson J. Activated synovial macrophages as targets for osteoarthritis drug therapy. Curr Drug Targets 2010;11:576-85.

5. Stöger JL, Goossens P, de Winther MP. Macrophage heterogeneity: relevance and functional implications in atherosclerosis. Curr Vasc Pharmacol 2010;8:233-48.

6. Woollard KJ, Geissmann F. Monocytes in atherosclerosis: subsets and functions. Nat Rev Cardiol 2010;7:77-86.

7. Suarez CJ, Parker NJ, Finn PW. Innate immune mechanism in allergic asthma. Curr Allergy Asthma Rep 2008;8.451-9.

8. Fairweather D, Cihakova D. Alternatively activated macrophages in infection and autoimmunity. J Autoimmun 2009;33:222-30.

9. Pieters J. Mycobacterium tuberculosis and the macrophage: maintaining a balance. Cell Host Microbe 2008;3:399-407.

10. Martins M, Viveiros M, Couto I, Amaral L. Targeting human macrophages for enhanced killing of intracellular XDR-TB and MDR-TB. Int J Tuberc Lung Dis 2009; 13:569-73.

11. Owais M, Gupta CM. Targeted drug delivery to macrophages in parasitic infections. Curr Drug Deliv 2005,2:311-18.

12. Frézard F, Demicheli C. New delivery strategies for the old pentavalent antimonial drugs. Expert Opin Drug Deliv 2010; 7:1343-58.

13. Coiras M, López-Huertas MR, Pérez-Olmeda M, Alcamí J. Understanding HIV-1 latency provides clues for the eradication of longterm reservoirs. Nat Rev Microbiol 2009;7: 798-812.

14. Gunaseelan S, Gunaseelan K, Deshmukh M, et al. Surface modifications of nanocarriers for effective intracellular delivery of anti-HIV drugs. Adv Drug Deliv Rev 2010; 62:518-31.

15. Herbein G, Varin A. The macrophage in HIV1 infection: from activation to deactivation? Retrovirology 2010;7:33-48.

16. Gavegnano C, Schinazi RF. Antiretroviral therapy in macrophages: implication for HIV eradication. Antivir Chem Chemother 2009; 20:63-78.

17. Qian BZ, Pollard JW. Macrophage diversity enhances tumor progression and metastasis. Cell 2010;141:39-51.

18. Siveen KS, Kuttan G. Role of macrophages in tumour progression. Immunol Lett 2009;123:97-102.

19. Solinas G, Germano G, Mantovani A, Allavena P. Tumor-associated macrophages (TAM) as 
major players of the cancer-related inflammation. J Leukoc Biol 2009;86:1065-73.

20. Chellat F, Merhi Y, Moreau A, Yahia L. Therapeutic potential of nanoparticulate systems for macrophage targeting. Biomaterials 2005;26:7260-75.

21. Torchilin VP. Recent advances with liposomes as pharmaceutical carriers. Nat Rev Drug Discov 2005;4:145-60.

22. Ahsan F, Rivas IP, Khan MA, Torres Suarez AI. Targeting to macrophages: role of physicochemical properties of particulate carriersliposomes and microspheres-on the phagocytosis by macrophages. J Control Release 2002;79:29-40.

23. van Rooijen N, Hendrikx E. Liposomes for specific depletion of macrophages from organs and tissues. Meth Mol Biol 2010; 605: 189-203.

24. Zeisberger SM, Odermatt B, Marty C, et al. Clodronate-liposome-mediated depletion of tumour-associated macrophages: a new and highly effective antiangiogenic therapy approach. Br J Cancer 2006;95:272-81.

25. Schwendener RA. Liposomes in biology and medicine. Adv Exp Med Biol 2007;620:117-28.

26. Seiler P, Aichele P, Odermatt B, et al. Crucial role of marginal zone macrophages and marginal zone metallophils in the clearance of lymphocytic choriomeningitis virus infection. Eur J Immunol 1997;27:2626-33.

27. Wassef NM, Alving CR. Complement-dependent phagocytosis of liposomes. Chem Phys Lipids 1993;64:239-48.

28. Devine DV, Wong K, Serrano K, et al. Liposome-complement interactions in rat serum: implications for liposome survival studies. Biochim Biophys Acta 1994;1191:4351.

29. Cullis PR, Chonn A, Semple SC. Interactions of liposomes and lipid-based carrier systems with blood proteins: Relation to clearance behaviour in vivo. Adv Drug Deliv Rev 1998; 32:3-17.

30. Bradley AJ, Devine DV. The complement system in liposome clearance: Can complement deposition be inhibited? Adv Drug Deliv Rev 1998;32:19-29.

31. Moghimi SM, Hunter AC. Recognition by macrophages and liver cells of opsonized phospholipid vesicles and phospholipid headgroups. Pharm Res 2001;18:1-8.

32. van Lookeren Campagne M, Wiesmann C, Brown EJ. Macrophage complement receptors and pathogen clearance. Cell Microbiol 2007;9:2095-102.

33. Andersen AJ, Hashemi SH, Andresen TL, et al. Complement: alive and kicking nanomedicines. J Biomed Nanotechnol 2009;5: 364-72.

34. Schwendener RA, Trüb T, Schott $\mathrm{H}$, et al. Comparative studies of the preparation of immunoliposomes with the use of two bifunctional coupling agents and investigation of in vitro immunoliposome-target cell binding by cytofluorometry and electron microscopy. Biochim Biophys Acta 1990;1026:69-79.

35. Duncan RJ, Weston PD, Wrigglesworth R. A new reagent which may be used to introduce sulfhydryl groups into proteins, and its use in the preparation of conjugates for immunoassay. Anal Biochem 1983;132:68-73.

36. Marty C, Schwendener RA. Cytotoxic tumor targeting with scFv antibody-modified liposomes. Methods Mol Med 2005;109:389-402.

37. O'Brien J, Wilson I, Orton T, Pognan F. Investigation of the Alamar Blue (resazurin) fluorescent dye for the assessment of mammalian cell cytotoxicity. Eur J Biochem 2000; 267:5421-26.

38. Woodle MC, Newman MS, Cohen JA. Sterically stabilized liposomes: physical and biological properties. J Drug Target 1994;2:397403.

39. He XM, Carter DC. Atomic structure and chemistry of human serum albumin. Nature 1992;358:209-15.

40. Quinlan GJ, Martin GS, Evans TW. Albumin: biochemical properties and therapeutic potential. Hepatology 2005;41:1211-19.

41. Zhang F, Skoda MW, Jacobs RM, et al. Protein interactions studied by SAXS: effect of ionic strength and protein concentration for BSA in aqueous solutions. J Phys Chem B 2007; 111:251-59.

42. Thöle M, Nobmann S, Huwyler $\mathrm{J}$, et al. Uptake of cationzied albumin coupled liposomes by cultured porcine brain microvessel endothelial cells and intact brain capillaries. J Drug Target 2002;10:337-44.

43. Allen TM, Austin GA, Chonn A, et al. Uptake of liposomes by cultured mouse bone marrow macrophages: influence of liposome composition and size. Biochim Biophys Acta 1991;1061:56-64.

44. Allen TM. Long-circulating (sterically stabilized) liposomes for targeted drug delivery. Trends Pharmacol Sci 1994;15:215-20.

45. Du H, Chandaroy P, Hui SW. Grafted poly(ethylene glycol) on lipid surfaces inhibits protein adsorption and cell adhesion. Biochim Biophys Acta 1997;1326:236-48.

46. Claassen E. Post-formation fluorescent labelling of liposomal membranes. In vivo detection, localisation and kinetics. J Immunol Methods 1992;147:231-40.

47. Shmeeda H, Amitay Y, Gorin J, et al. Delivery of zoledronic acid encapsulated in folate-targeted liposome results in potent in vitro cytotoxic activity on tumor cells. J Controlled Release 2010;146:76-83.

48. Schwendener RA, Lagocki PA, Rahman YE. The effects of charge and size on the interaction of unilamellar liposomes with macrophages. Biochim Biophys Acta 1984;772:93101.

49. Schroit AJ, Madsen J, Nayar R. Liposome-cell interactions: in vitro discrimination of up- take mechanism and in vivo targeting strategies to mononuclear phagocytes. Chem Phys Lipids 1986;40:373-93.

50. Opanasopit P, Higuchi Y, Kawakami S, et al. Involvement of serum mannan binding proteins and mannose receptors in uptake of mannosylated liposomes by macrophages. Biochim Biophys Acta 2001;1511: 134-45.

51. Vyas SP, Goyal AK, Khatri K. Mannosylated liposomes for targeted vaccines delivery. Methods Mol Biol 2010;605:177-88.

52. Dubey V, Nahar M, Mishra D, et al. Surface structured liposomes for site specific delivery of an antiviral agent-indinavir. J Drug Target 2011;19:258-69.

53. Paulos CM, Turk MJ, Breur GJ, Low PS. Folate receptor-mediated targeting of therapeutic and imaging agents to activated macrophages in rheumatoid arthritis. Adv Drug Deliv Rev 2004;56:1205-17.

54. Rensen PC, Gras JC, Lindfors EK, et al. Selective targeting of liposomes to macrophages using a ligand with high affinity for the macrophage scavenger receptor class A. Curr Drug Discov Technol 2006;3:135-44.

55. Sou K, Goins B, Takeoka S, et al. Selective uptake of surface-modified phospholipid vesicles by bone marrow macrophages in vivo. Biomaterials 2007;28:2655-66.

56. Chonn A, Cullis PR, Devine DV. The role of surface charge in the activation of the classical and alternative pathways of complement by liposomes. J Immunol 1991;146:4234-41.

57. Harashima H, Ochi Y, Kiwada H. Kinetic modelling of liposome degradation in serum: effect of size and concentration of liposomes in vitro. Biopharm Drug Dispos 1994;15:21725.

58. Furumoto K, Yokoe J, Ogawara K, et al. Effect of coupling of albumin onto surface of PEG liposome on its in vivo disposition. Int $\mathrm{J}$ Pharm 2007;329:110-16.

59. Watanabe M, Kawano K, Toma K, et al. In vivo antitumor activity of camptothecin incorporated in liposomes formulated with an artificial lipid and human serum albumin. J Control Release 2008;127:231-38.

60. Aharinejad S, Sioud M, Lucas T, Abraham D. Targeting stromal-cancer cell interactions with siRNAs. Methods Mol Biol 2009;487:24366.

61. Brynskikh AM, Zhao Y, Mosley RL, et al. Macrophage delivery of therapeutic nanozymes in a murine model of Parkinson's disease. Nanomedicine 2010;5:379-96.

62. Vasievich EA, Huang L. The suppressive tumor microenvironment: a challenge in cancer immunotherapy. Mol Pharm 2011;8:63541.

63. Sayed D, Abdellatif M. MicroRNAs in Development and Disease. Physiol Rev 2011;91:82787. 